\title{
Development and Integration of the CT-PPS Fast Simulation in the CMS Software
}

\author{
Sandro Fonseca De Souza on behalf of the CMS and TOTEM Collaborations* \\ Universidade do Estado do Rio de Janeiro (BR) \\ E-mail: sandro.fonseca@cern.ch
}

\begin{abstract}
CT-PPS (CMS-TOTEM Precision Proton Spectrometer) is a joint project of the CMS and TOTEM collaborations with the goal of studying central exclusive production (CEP) in proton-proton collisions. A simplified simulation and reconstruction code for CT-PPS has been implemented in the CMS fast simulation package FastSim. Protons scattered at very low polar angles are propagated along the LHC beamlines from the generated vertex to the detectors by means of the beam transport package Hector. The reconstructed proton tracks are obtained from the simulated hits in the tracking detectors and are used to determine the proton kinematics at the vertex. The timing information is added to the tracks.
\end{abstract}

The European Physical Society Conference on High Energy Physics

5-12 July

Venice, Italy

${ }^{*}$ Speaker. 


\section{The CT-PPS Detector}

CT-PPS (CMS-TOTEM Precision Proton Spectrometer) [1] is a joint project the CMS [2] and TOTEM [3] collaborations with the goal of studying central exclusive production (CEP) in proton-proton collisions. The aim is to study processes mediated by colourless exchanges with large rapidity gaps in the final state, like photon-photon and gluon-gluon fusion, as can be seen in Figure 1, where the high $p_{T}$ system is detected by the central CMS detectors and the scattered protons are detected by CT-PPS.
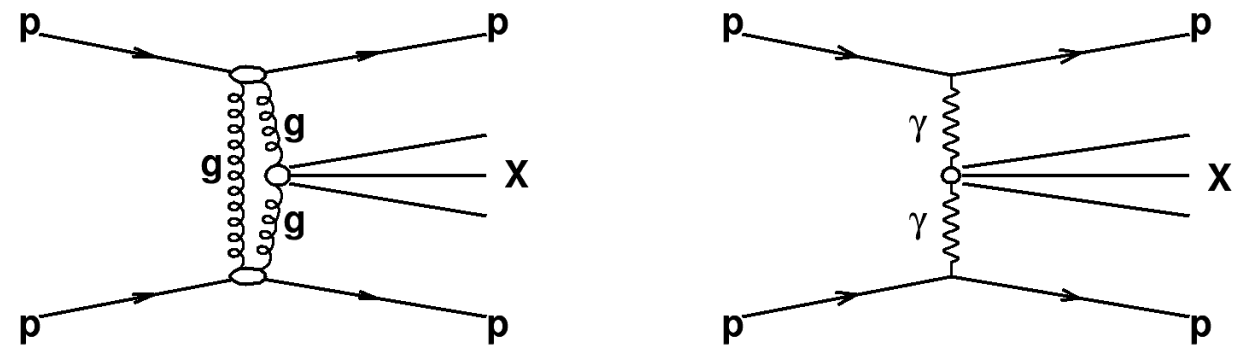

Figure 1: CEP from gluon-gluon (left) and photon-photon (right) fusion

The four-momentum of the central system is fully constrained by the protons kinematics, namely the proton fractional momentum loss $\xi$ and the squared four-momentum transfer at the proton vertex $t$, which can be obtained from the hits in CT-PPS and the LHC optics. CT-PPS has several components: two stations of silicon tracker detectors to measure the proton momentum and one station of timing counters to disentangle the pile-up vertices. They are housed in the horizontal TOTEM Roman Pots, located between 200m and 220m at each side of the CMS IP. Specialized cylindrical Roman Pots were built for the time of flight detector. The layout of CT-PPS is shown in Figure 2.

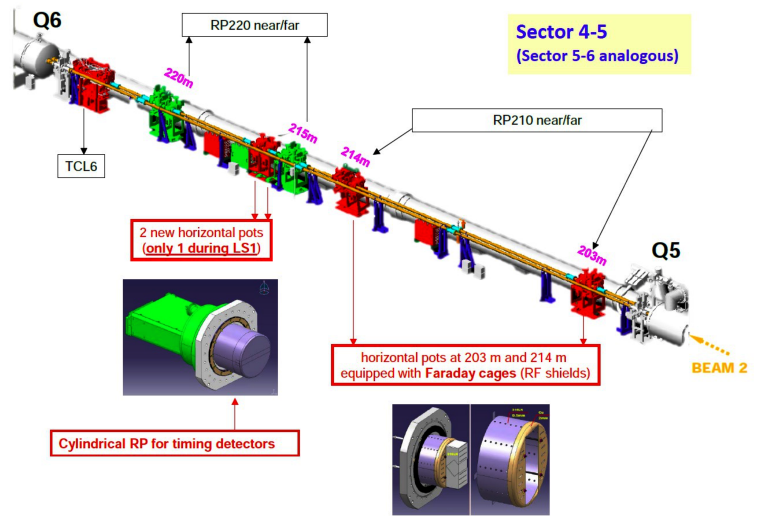

Figure 2: Schematic layout of CT-PPS

\section{FastSim for CT-PPS}

The fast simulation of the CMS detector (henceforth FastSim) [4] is an object-oriented system, written in $\mathrm{C}++$ and steered by Python configuration files, in the CMS software framework 
Development and Integration of the CT-PPS Fast Simulation in the CMS Software

Sandro Fonseca De Souza on behalf of the CMS and TOTEM Collaborations

(CMSSW) [5]. It is alternative and complementary to the Geant4-based approach (for which we employ the term "full simulation", including the electronic effects simulated by CMSSW-specific modules), with respect to which it is regularly validated and tuned. The block diagram shown in Figure 3 represents the CT-PPS FastSim modules integrated in CMS FastSim. For the tracking detectors, both the TOTEM Si strips and the 3D pixel sensors [6] can be simulated. For the timing, diamond sensors [7] and Ultra Fast Silicon Detectors [8] are available.

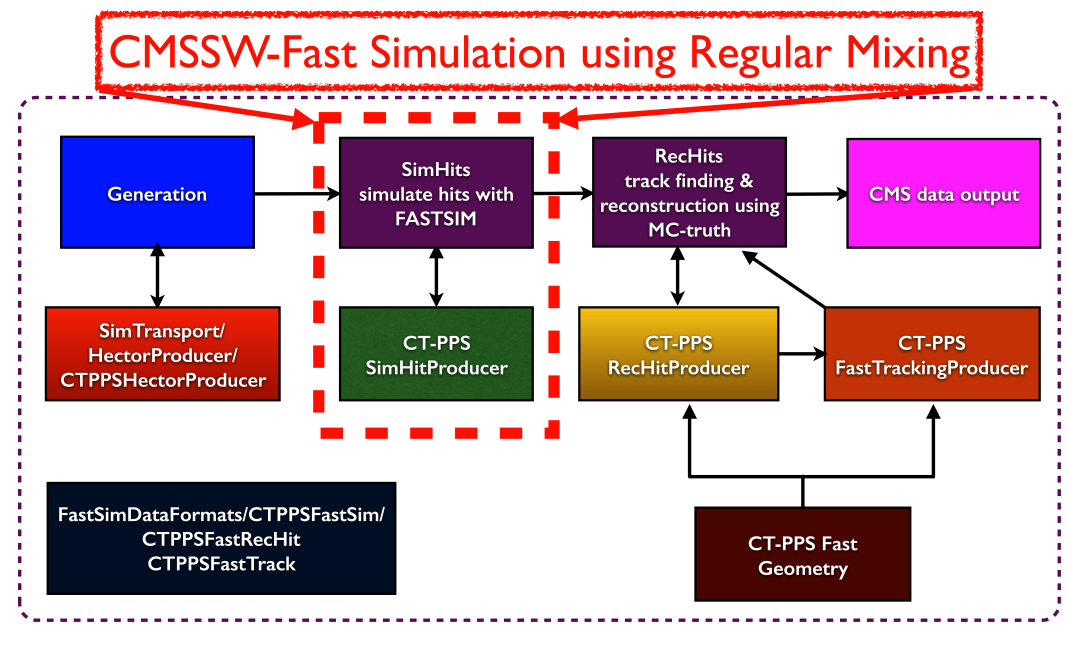

Figure 3: Block diagram of the FastSim code for CT-PPS. The code is officially integrated and validated in the CMS Software (CMSSW).

\section{Track reconstruction}

The SimTransport package in CMSSW is an interface to Hector [9], which uses transfer matrices to propagate single particles through the magnetic elements of the accelerator (dipoles and quadrupoles) and other elements like collimators and aperture limitations. This package is used both for transporting the generated protons up to the location of the detectors and for inferring the kinematical variables at the collision point, allowing to determine the protons momenta, as well as $t$ and $\xi$.

\section{A physics example: ExHuME gg $\rightarrow$ jj}

Exclusive dijet production (Figure 1, left) is one of the interesting processes that can be studied by measuring the jets in the central part of CMS and the scattered protons in CT-PPS. The ExHuME generator [10] interfaced to PYTHIA was used to simulate events for this channel at $\sqrt{s}=13 \mathrm{TeV}$, with dijet invariant masses between 300 and $2000 \mathrm{GeV}$. The reconstructed hit maps are shown in Figure 4. In this simulation, the detectors are at a distance of $15 \sigma$ from the beam centre, with $\sigma$ the standard deviation of the spatial distribution of the beam in the transverse plane. An LHC optics with $\beta^{*}=0.6 \mathrm{~m}$ and crossing angle $142.5 \mu \mathrm{rad}$ is assumed. 

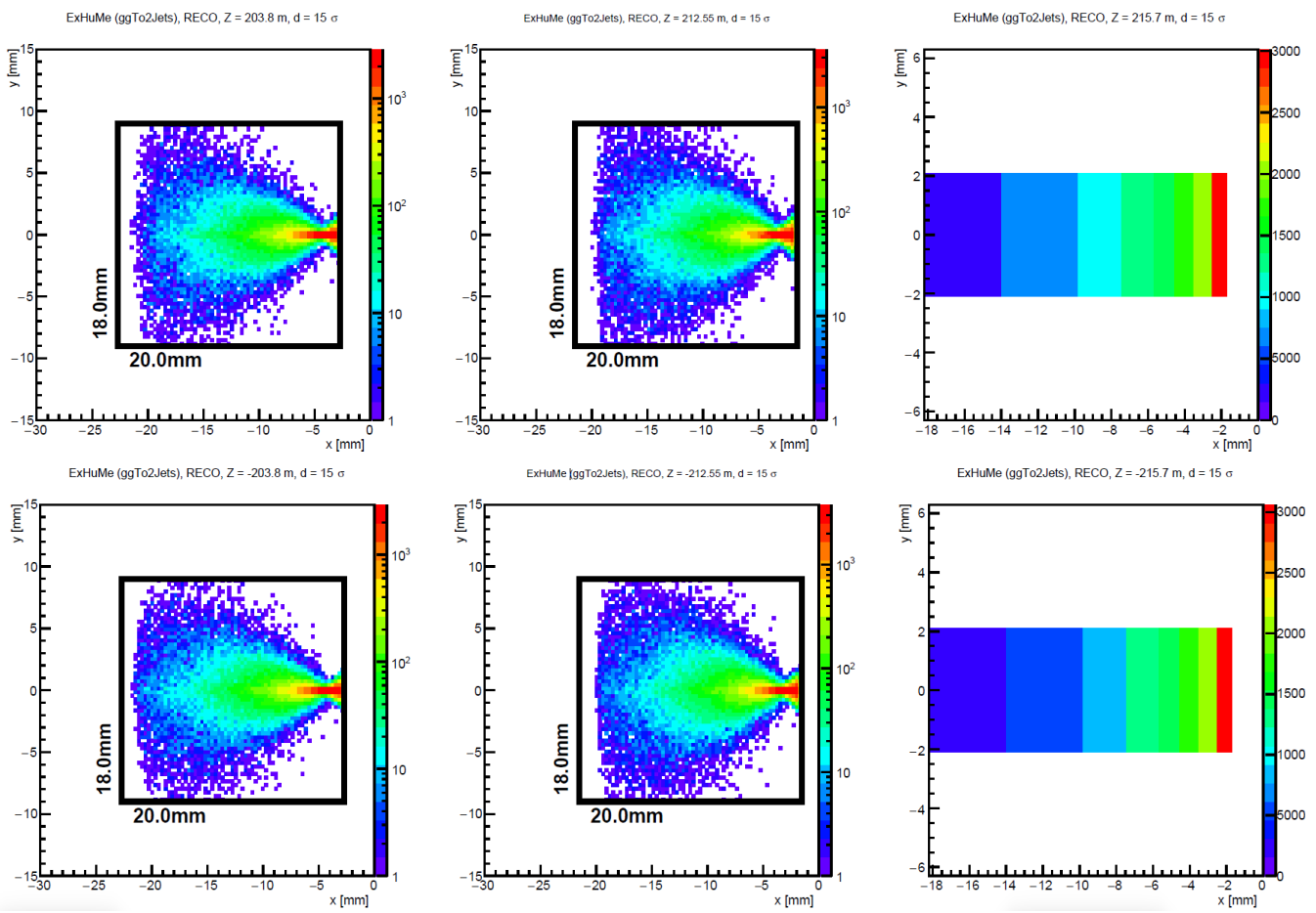

Figure 4: Reconstructed hit maps for events generated with ExHuME. The tracking and timing (rightmost column) detectors are shown.

\section{References}

[1] The CMS and TOTEM Collaborations, "CMS-TOTEM Precision Proton Spectrometer Technical Design Report", CERN-LHCC-2014-021, CMS-TDR-13, TOTEM-TDR-003.

[2] The CMS Collaboration, "The CMS experiment at the CERN LHC", JINST 3 S08004 (2008).

[3] The TOTEM Collaboration, "The TOTEM Experiment at the CERN Large Hadron Collider", JINST 3 S08007 (2008).

[4] A. Giammanco, R. Kroeger, and R. Rahmat, "The fast simulation of the CMS experiment", J.Phys.Conf.Ser.,396:062016, (2012).

[5] CMS Collaboration. "CMS technical design report, volume I: Detector performance and software", Technical Report CERN-LHCC-2006-001, CERN, (2006).

[6] A. Solano, "The CT-PPS tracking system with 3D pixel detectors", in $12^{\text {th }}$ Trento Workshop on Advanced Silicon Radiation Detectors (20-22 February 2017).

[7] M. Berretti, The TOTEM Collaboration, "The timing upgrade project of the TOTEM Roman Pots detectors", NIM A824 (2016) 251-252.

[8] N. Cartiglia et al., "Performance of ultra-fast silicon detectors", JINST 9 C02001 (2014).

[9] X. Rouby, J. de Favereau, K. Piotrzkowski, "Hector, a fast simulator for the transport of particles in beamlines", JINST 2 P09005 (2007).

[10] J. Monk and A. Pilkington, "ExHuME: A Monte Carlo event generator for exclusive diffraction", Comput. Phys. Commun. 175, 232 (2006) [hep-ph/052077]. 